Documentation et bibliothèques

\title{
La Bibliothèque municipale de Montréal : survol historique et perspectives d'avenir
}

\section{Jacques Panneton}

Volume 34, numéro 1, janvier-mars 1988

URI : https://id.erudit.org/iderudit/1052547ar

DOI : https://doi.org/10.7202/1052547ar

Aller au sommaire du numéro

\section{Éditeur(s)}

Association pour l'avancement des sciences et des techniques de la

documentation (ASTED)

\section{ISSN}

0315-2340 (imprimé)

2291-8949 (numérique)

Découvrir la revue

\section{Citer cet article}

Panneton, J. (1988). La Bibliothèque municipale de Montréal : survol historique et perspectives d'avenir. Documentation et bibliothèques, 34(1), 19-24.

https://doi.org/10.7202/1052547ar

Tous droits réservés ( $)$ Association pour l'avancement des sciences et des techniques de la documentation (ASTED), 1988
Ce document est protégé par la loi sur le droit d'auteur. L'utilisation des services d'Érudit (y compris la reproduction) est assujettie à sa politique d'utilisation que vous pouvez consulter en ligne.

https://apropos.erudit.org/fr/usagers/politique-dutilisation/ 


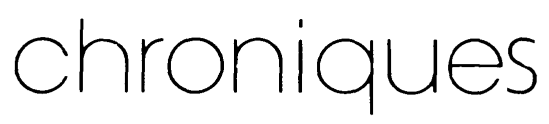

\section{La Bibliothèque municipale de Montréal: survol historique et perspectives d'avenir}

La Bibliothèque municipale de Montréal représente une grande richesse collective, un acquis important dans le monde documentaire au Québec. À maints égards, la Bibliothèque de Montréal occupe une place privilégiée dans I'histoire québécoise de l'information documentaire. Ayant été crée en 1902, elle fut le témoin et garde la mémoire de l'évolution de la société montréalaise depuis le début du siècle, et même avant. La Bibliothèque de Montréal a contribué substantiellement à la protection et à la conservation du patrimoine du Québec grâce à l'acquisition en 1910, puis au développement subséquent de la collection du bibliophile Philéas Gagnon. Ceci avant même que la bibliothèque Saint-Sulpice n'existât et qu'elle ne devînt en 1967 la Bibliothèque nationale.

\section{Survol historique}

En 1902, les autorités municipales de Montréal adoptent un premier règlement concernant l'établissement d'une bibliothèque publique et gratuite. L'année suivante, principalement sous l'impulsion de la Chambre de Commerce et de I'Association Saint-Jean-Baptiste, une bibliothèque scientifique et industrielle est mise sur pied surtout pour répondre aux besoins des travailleurs.

Logée au départ dans une petite pièce du Monument national, cette bibliothèque croît au point de nécessiter un déménagement dans l'édifice de l'École technique, rue Sherbrooke.

Une dizaine d'années plus tard, grâce à la ténacité du conseiller municipal Victor Morin et à ses appuis dans le public, la Ville de Montréal décide de consacrer près d'un demi-million de dollars à la construction d'un édifice digne de loger la bibliothèque de la métropole du Canada.

Les travaux commencent en 1915 et la nouvelle bibliothèque est mise en service en 1917. II s'agit de l'immeuble logeant actuellement la bibliothèque centrale, rue Sherbrooke. Cet édifice de style Beaux-Arts est l'oeuvre de l'architecte Eugène Payette, auteur également de la Bibliothèque Saint-Sulpice. Ces deux édifices se révèlent des éléments importants du patrimoine architectural de Montréal.

Le 28 mai 1917, le Conseil municipal adopte le règlement 624 concernant l'établissement et l'administration d'une bibliothèque publique:

Une bibliothèque publique et gratuite est établie dans la ville de Montréal pour propager dans le public la connaissance des sciences, des arts et de la bonne littérature. Elle contiendra tous les livres, publications, recueils, journaux, revues et dessins propres à atteindre ce but...

La mise en service de la nouvelle bibliothèque permet également de rendre disponible à la consultation, au début des années 1920, une collection prestigieuse acquise dix années plus tôt par la Ville, à savoir la bibliothèque personnelle du bibliophile québécois Philéas Gagnon. Cette collection est à l'époque et reste encore aujourd'hui une des plus remarquables collections de Laurentiana au monde.

\section{Les bibliothèques de quartier}

La Bibliothèque de Montréal a également été un instrument de diffusion et un agent de développement de la communauté par l'établissement de plusieurs succursales dans le territoire de la Ville dès après la seconde guerre. À partir de 1947 , on inaugure les premières bibliothèques de quartier: Shamrock, qui deviendra la bibliothèque Le Prévost en 1983; Workman, qui deviendra la bibliothèque Georges-Vanier en 1985; Hochelaga, qui deviendra la bibliothèque Sainte-Marie en 1970; de Lorimier; Rosemont; Ahuntsic. 
Depuis 1976 en particulier, la Ville a appliqué un programme majeur de révision et de réévaluation de ses équipements et de ses ressources centré sur les bibliothèques de quartier. Les citoyens et les citoyennes de tous les quartiers ont bénéficié de services améliorés:

ouverture de la bibliothèque Langelier (1980);

ouverture des bibliothèques du Métro McGill et de Maisonneuve, puis rénovation de la bibliothèque de l'Acadie (1981);

ouverture de la bibliothèque Mile-End et rénovation de l'ancienne bibliothèque Monk, connue maintenant sous le nom de la bibliothèque Marie-Uguay (1982);

construction de la bibliothèque Côte-des-Neiges et ouverture de la bibliothèque Le Prévost (1983);

- ouverture d'une annexe à la Bibliothèque centrale, de la bibliothèque du Plateau MontRoyal et de la bibliothèque Notre-Dame-deGrâce (1984);

- aménagement de la Phonothèque de prêt et rénovation de la bibliothèque Georges-Vanier (1985);

- rénovation de la bibliothèque De Lorimier, maintenant dénommée la Petite Patrie (1987);

- mise en chantier des bibliothèques Mercier et Parc Frontenac (1987)

Le territoire de Montréal est donc entièrement couvert par son réseau de bibliothèques, exception faite du quartier Rivière-des-Prairies qui fait l'objet d'une étude à ce moment-ci.

\section{Ressources actuelles du réseau}

Le parti pris d'action de la Ville a été récemment réaffirmé de façon dynamique par les dirigeants municipaux devant la Commission d'étude sur les bibliothèques publiques dont le rapport a été déposé en juillet 1987. Citons ici de courts extraits du mémoire de la Ville de Montréal, manifestant bien l'importance accordée au développent des bibliothèques:

Dans le but de satisfaire les besoins d'information, d'éducation, de culture et de loisir intellectuel des individus et des groupes, la
Bibliothèque de Montréal rassemble avec objectivité et rend disponible à tous, sans discrimination, une documentation variée constituée de livres, brochures, périodiques, autres imprimés, banques de données, films, vidéo-cassettes, enregistrements sonores et autres. Cette documentation est organisée systématiquement de manière à assurer l'accessibilité intellectuelle; de plus, la bibliothèque stimule et facilite aux citoyens I'utilisation de la documentation par l'apport d'un personnel de qualité, d'un service d'interprétation des collections, de services d'animation et de mise en valeur des ressources documentaires ${ }^{2}$.

La Ville affirmait aussi que "l'information documentaire est un bien public indispensable à l'évolution de la société et que la bibliothèque publique est l'institution démocratique la mieux adaptée pour en assurer l'accès à tous les individus qui la composent». Enfin, «la Ville de Montréal considère que c'est une responsabilité politique inaliénable que de créer et de maintenir les conditions qui permettent la réalisation de ce projet».

Dans l'action, ces déclarations correspondent à une réalité. Pour rencontrer ce programme, la Ville de Montréal propose en effet une offre de service substantielle. L'entité principale du réseau est la Bibliothèque centrale. Elle est axée surtout sur l'information, la gestion et la conservation des fonds documentaires dans une perspective à long terme. Elle sert d'appui et de bibliothèque de ressources à toutes les bibliothèques de quartier. Elle offre un service de prêt et de consultation à raison de 60 heures par semaine: prêt et consultation de documents, prêt entre bibliothèques et réponse aux questions de référence soit sur place, soit par téléphone, soit par courrier. De plus, elle dispense un service de téléréférence qui permet aux usagers d'accéder à la consultation de banques de données.

La Bibliothèque centrale est reliée à un réseau de 23 bibliothèques de quartier dont huit sont situées dans des maisons de la culture. Le réseau des bibliothèques de quartier fonctionne à raison de 35 heures par semaine et offre un service de consultation et de prêt de documents. La demande documentaire des usagers est orientée surtout vers les parutions récentes, le livre pratique et le roman.

Les collections des bibliothèques de quartier ont été enrichies avec des éléments audiovisuels: postes d'écoute de musique, collections de 


\section{Tableau 1}

\section{Données statistiques sur le réseau de la} Bibliothèque municipale de Montréal

NOMBRE DE BIBLIOTHĖQUES

BIBLIOBUS (23 arrêts)

SERVICES EXTÉRIEURS

Institutions desservies

BIBLIOSERVICE MULTILINGUE

Nombre de bibliothèques partipantes

au Québec

du réseau

SUPERFICIE DES LOCAUX

Bibliothèque principale

Succursales

NOMBRE DE PLACES ASSISES

Bibliothèque principale

HEURES D'OUVERTURE (Hiver-Moyenne)

- Bibliothèque Centrale - Adultes

- Cinémathèque et Phonothèque

- Succursales Adultes

- Succursales Enfants

PERSONNEL

- Bibliothécaires

- Techniciens

65,5

- Employés de soutien

Total

BUDGET (dépenses)

\section{FRÉQUENTATION DE LA BIBLIOTHĖQUE}

Prêts externes

Prêts entre bibliothèques

- Documents empruntés 


\section{Tableau 2}

Ressources documentaires ${ }^{1}$

Bibliothèque municipale de Montréal

ÉTAT DES COLLECTIONS

ACQUISITIONS DE LIVRES ET BROCHURES

56209

INVENTAIRE DE LIVRES ET BROCHURES

Adultes

Enfants

1638930

531347

- Total

2170277

REVUES ET JOURNAUX

Collections courantes de revues

Titres

- Abonnements

Collections courantes de journaux

- Titres

Abonnements

Nombre total de titres

- Collections courantes et non courantes de revues

- Collections courantes et non courantes de journaux

PUBLICATIONS OFFICIELLES

MICRODOCUMENTS (unités physiques)

147476

DOCUMENTS AUDIOVISUELS

DOCUMENTS DE DIVERSES CATÉGORIES

Cartes géographiques

4925

Photographies

47534

Collections théâtre

2848

Timbres

1930

Monnaies

698

Cartons d'archives

874

Dons

6518

Collection Duquesne

3017

571

Guides de voyage

1909

Fonds documentaire

1125

Partitions musicales

3593

Posters

3939

Brochures

1 Source: Enquête annuelle du ministère des Affaires culturelles, 1986. 
cassettes pour le prêt à domicile, cours de langues passifs et participatifs, services de visionnement de vidéos. En plus des bibliothèques de quartier, la Bibliothèque municipale de Montréal offre un service audiovisuel central à la Cinémathèque et à la Phonothèque. À cet endroit, la Ville dispose d'une ressource majeure qui offre les mêmes types de services audiovisuels que les bibliothèques de quartier, plus la location de films, de diapositives et d'équipements de projection, le visionnement sur place de films et de vidéos, des collections importantes de disques et de cassettes tant pour le prêt à domicile que pour l'écoute sur place.

Certains quartiers de la ville sont desservis par le bibliobus; soit, les secteurs périphériques qui ne possèdent pas encore de bibliothèques, et les secteurs à faible densité de population pour lesquels l'implantation d'une bibliothèque représente, pour le moment, une difficulté économique réelle. Le bibliobus visite chacun de ces endroits toutes les deux semaines.

La Bibliothèque municipale de Montréal a également développé des services documentaires dans la langue d'origine des communautés culturelles montréalaises. Elle le fait de deux manières: premièrement, en acquérant ses propres collections. Elles sont localisées principalement à la bibliothèque du Mile-End, où l'on trouve des collections en espagnol, en grec, en portugais, en vietnamien et en italien, à la bibliothèque Côte-des-Neiges (espagnol et vietnamien), et à la bibliothèque Mont-Royal (espagnol et portugais).

Deuxièmement, ce service est complété par la collection du biblioservice multilingue de la Bibliothèque nationale du Canada. Ce service dépose à la Bibliothèque de Montréal des collections dans une vingtaine de langues. Ce fond, géré par la Bibliothèque municipale de Montréal, est utilisé dans l'ensemble des bibliothèques québécoises mais également par la Bibliothèque de Montréal elle-même, pour compléter dans d'autres langues les fonds documentaires dont la bibliothèque n'a pas pu se doter elle-même.

La Bibliothèque municipale de Montréal offre également un certain nombre de services adaptés qui permettent d'atteindre des clientèles particulières qu'elle n'arriverait pas à rejoindre normalement par son réseau. Songeons en particulier au prêt de collections en institution: des livres sont déposés dans des établissements pour personnes âgées, ou dans des centres hospitaliers de soins prolongés, où l'on trouve évidemment des usagers privés de mobilité. Songeons également aux gens retenus à domicile qui sont rejoints par le service Biblio-courrier, qui permet de leur livrer des livres à la maison. La Biblio- thèque municipale de Montréal prête également des livres enregistrés sur cassettes pour les nonvoyants et les faibles voyants.

\section{Programme d'animation}

Ces différents services sont appuyés au niveau des bibliothèques de quartier par un programme d'animation. La Bibliothèque y offre des activités dont la fonction est premièrement d'élargir la variété de l'offre culturelle et, deuxièmement, de présenter des événements qui permettent la mise en valeur des ressources documentaires en fonction des centres d'intérêt divers.

Cet important programme représente environ 4 000 activités chaque année dans l'ensemble des bibliothèques et couvre des domaines qui vont du cinéma à la musique, des marionnettes au théâtre, des ateliers de lecture aux ateliers d'écriture. II comprend également des ateliers d'information qui traitent de questions comme les finances, l'écologie, le loisir et la santé de manière à inscrire la bibliothèque au coeur des préoccupations de ses usagers.

\section{Une institution ouverte sur l'avenir}

La Bibliothèque municipale de Montréal, c'est enfin une institution ouverte sur un réseau documentaire beaucoup plus large qui permet aux citoyens et aux citoyennes de Montréal d'accéder à l'ensemble des ressources disponibles. Pour ce faire, elle maintient des rapports de collaboration avec les associations professionnelles actives dans le domaine de la documentation, avec les grandes institutions documentaires comme la Bibliothèque nationale du Canada, la Bibliothèque nationale du Québec, les bibliothèques d'universités, de même qu'avec les écoles de bibliothéconomie et des sciences de l'information.

Le budget de 1988 permet de maintenir intégralement la structure de service offerte en 1987. en plus d'ajouter de nouvelles activités résumées ici:

mise en service, à l'hiver 1988-1989, des nouvelles bibliothèques Mercier et Parc Frontenac, présentement en construction;

augmentation de l'achat de documents courants à la Bibliothèque du Mile-End à l'intention des communautés culturelles grecque, vietnamienne, espagnole, portugaise, italienne, créole et chinoise: livres, périodiques, vidéo-cassettes, cassettes audio;

établissement d'un centre de ressources documentaires, dans le but de regrouper, gérer et exploiter les documents semi-actifs. Les 
activités de 1988 concernent le choix des documents à transférer, leur relogement et leur organisation. Plus de 80000 documents sont visés à court terme.

Enfin, signalons que 1988 verra le début de l'automatisation de la Bibliothèque municipale de
Montréal par la confirmation du choix du logiciel et de l'infrastructure informatique.

Jacques Panneton*

Bibliothèque municipale de Montréal

* L'auteur de cet article est Assistant directeur au service des Loisirs et du développement communautaire, et Bibliothécaire en chef de la Ville de Montréal.

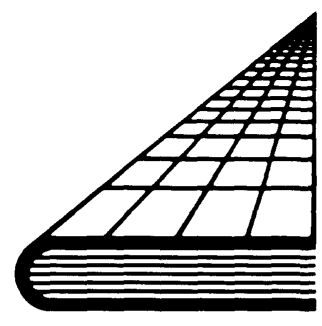

âitre son environnement, c'est mieux gérer son avenir et son développement professionnel. Cette année, nous vous proposons d'atteindre vos objectifs en rencontrant de nombreux spécialistes de domaines aussi divers que la démographie, la sociologie, la politique, la gestion, les affaires culturelles et le développement technologique.

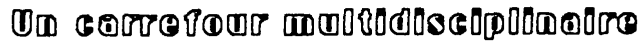

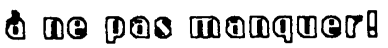

Pour Information: $\quad C B P Q$

307 ouest, rue Ste-Catherine Bureau 320

Montréal (Québec) H2X 2 A3

Tél: (514) 845-3327 